\title{
Optical temperature measurements on thin freestanding silicon membranes
}

\author{
Markus Schmotz, ${ }^{\text {a) }}$ Patrick Bookjans, Elke Scheer, and Paul Leiderer \\ University of Konstanz, 78457 Konstanz, Germany
}

(Received 30 June 2010; accepted 13 September 2010; published online 5 November 2010)

\begin{abstract}
We report on a contactless, all-optical method to derive thermal properties of $340 \mathrm{~nm}$ thin, 640 $\times 640 \mu \mathrm{m}^{2}$ wide, freestanding silicon membranes. Exploiting the temperature dependent optical transmissivity of $4 \times 10^{-3} / \mathrm{K}$ between 300 and $470 \mathrm{~K}$, we are able to measure the temperature of the membrane with millikelvin thermal, micrometer lateral, and nanosecond temporal resolution. Experiments such as the decay of a transient thermal grating with spacings between 5 and $30 \mu \mathrm{m}$, measured in first order of diffraction, as well as heat diffusion in a statically heated membrane are reported in this letter. For the latter case an example of a nanostructured membrane by means of focused ion beam milling is given. (C) 2010 American Institute of Physics. [doi:10.1063/1.3499503]
\end{abstract}

\section{INTRODUCTION}

Thermal properties of structures on small length scales are of crucial importance for many applications in microand nanotechnology. The thermal transport in systems such as silicon-on-insulator (SOI) circuits, novel threedimensional multilayer electronics, nano- or microelectromechanical systems with semiconducting or dielectric membranes is essential for the functioning of these devices. ${ }^{1}$ The investigation of the thermal properties of such small structures on the scale of micrometers and below requires special sensors, for which in addition to high spatial resolution often also a fast temporal resolution is required. Relevant time scales of thermal relaxation and transport processes in these systems can be as short as nanoseconds. We report here on a contactless method for deriving thermal properties of thin, freestanding $\mathrm{Si}$ membranes, which make it an interesting candidate for these tasks. Since the readout is done optically there is no need for any electrical contacts, which are often impedimental and delicate to prepare on small length scales.

The dielectric function of $\mathrm{Si}$ is known to be distinctly temperature dependent. ${ }^{2}$ The sensor principle described here is based on utilizing this dependence. As will be shown, the spatially resolved optical transmissivity of a $\mathrm{Si}$ membrane can be directly used to determine its local temperature profile and to obtain two-dimensional maps of the temperature distribution with micrometer and nanosecond resolution.

\section{PRINCIPLES}

The Si membranes were prepared from SOI wafers by the method of Butschke et al. ${ }^{3}$ with adaptions. ${ }^{4}$ They had a thickness of $340 \mathrm{~nm}$ and an area of approximately 640 $\times 640 \mu \mathrm{m}^{2}$, the exact size varying slightly from sample to sample. Figure 1(a) shows an optical transmission spectrum recorded in the range between 400 and $1000 \mathrm{~nm}$ for a set of four different temperatures, starting from 300 up to $470 \mathrm{~K}$. They exhibit characteristic Fabry-Perot-like interference oscillations typical for thin layers. When the temperature is

\footnotetext{
${ }^{a)}$ Electronic mail: markus.schmotz@uni-konstanz.de.
}

increased, the transmission is observed to drop in most parts of the spectrum, indicating an increase of the absorption coefficient. In addition the positions of the maxima and minima shift slightly, an effect of thermal expansion and a change in the real part of the refractive index. Figure 1(b) shows the temperature dependence of the transmissivity at a fixed wavelength of $488 \mathrm{~nm}$, measured with an Ar-ion probe laser beam. At this wavelength the temperature dependence is pronounced and nearly linear between room temperature and $470 \mathrm{~K}$, and hence the sensitivity is nearly constant. We therefore have used this wavelength in most of the measurements presented below.

The data in Fig. 1(a) are in good agreement with calculations (also plotted in this figure) for a membrane with the given thickness, using parameters for the dielectric function of Si taken from literature. ${ }^{5}$ The slight deviations might be caused by residual $\mathrm{SiO}_{2}$ and surface roughness, not taken into account in the calculations. The accuracy in determining temperature differences $\Delta T$ within the membrane on the basis of Fig. 1(b) was $0.1 \mathrm{~K}$, corresponding to a sensitivity of $4 \times 10^{-4}$. Having a random noise level around $10^{-3}$ simple dc averaging over a few cycles allows for a sufficient signal-tonoise ratio. This could be further improved by extensive averaging to an accuracy better than $1 \mathrm{mK}$.

\section{EXPERIMENTS}

In order to demonstrate the potential of this optical phenomenon for the investigation of thermal properties we consider as two examples in Figs. 2 and 3 the space- and time-dependent temperature distribution in a membrane: In the experimental setup used for Fig. 2 the heating laser $(\lambda=658 \mathrm{~nm}$, laser intensity $P=14.3 \mathrm{~mW}$, focus diameter $a \approx 15 \mu \mathrm{m})$ was focused to the center of the membrane; the probe laser $\left(\lambda=488 \mathrm{~nm}, P_{\text {probe }}=80 \mu \mathrm{W}, a_{\text {probe }}=10 \mu \mathrm{m}\right)$ was scanned across the membrane and the transmitted intensity was recorded with a photodiode.

Figure 2 shows the effect of local heating in the center of the freestanding area. If the $\mathrm{Si}$ is continuously heated in a spot much smaller than the size of the membrane, the system can be considered as quasitwo-dimensional for heat transfer. 


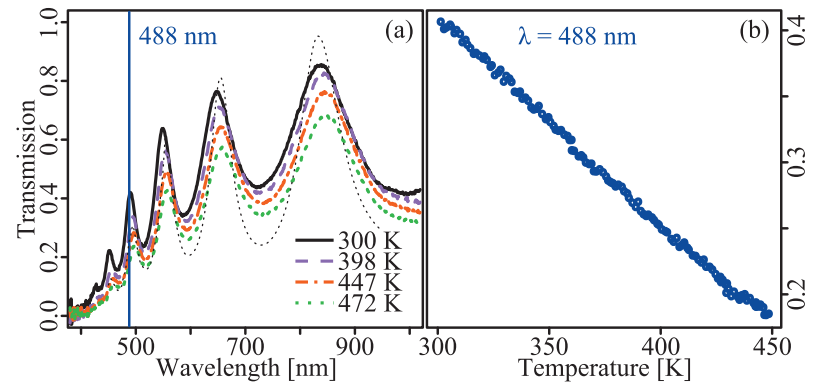

FIG. 1. (Color) (a) Transmission spectra of $340 \mathrm{~nm}$ thick membrane measured at various temperatures (see color code) and obtained by simulation for $300 \mathrm{~K}$ (dotted line). (b) Temperature dependent transmission measured at a given wavelength of $\lambda=488 \mathrm{~nm}$.

Under steady-state conditions the heat is continuously transferred from the hot center to the colder rim, where it is carried away by the surrounding wafer. This can now easily be measured exploiting the temperature dependence of the transmission, as shown previously, by varying the position of the probing spot; see Fig. 2. Solving Fourier's law in radialsymmetric geometry the temperature profile around the heated center should follow a logarithmic dependence

$$
T(r)=\frac{-P}{2 \pi \kappa d} \ln \frac{r}{r_{0}}+T_{0},
$$

where $P$ is the absorbed input power, $r$ the radial coordinate, $d$ the thickness of the membrane, $\kappa$ the thermal conductivity, $r_{0}$ and $T_{0}$ a starting radius and temperature, respectively. A comparison of the data with a fit according to Eq. (1) shows that the functional form agrees quite well with the expectation. Here we neglect the weak temperature dependence of $\kappa$. The absolute value of the thermal conductivity as derived from the fit in Fig. 2 is $\kappa=136 \pm 27 \mathrm{~W} / \mathrm{m} \mathrm{K}$, which is very close to the values quoted in literature. ${ }^{6}$ Additional contributions to thermal transport, which might come to mind, are negligible here: Assuming the temperature rise to be $200 \mathrm{~K}$ an area of $10^{-3} \mathrm{~mm}^{2}$ (heated by the laser spot) radiates $3.3 \mu \mathrm{W}$ of heat by Stefan-Boltzmann's law. Without any heat transfer within the membrane it would take more than $30 \mathrm{~ms}$ for the system to cool down by pure radiation. The experiments, however, showed that the time scales are in the nanosecond to microsecond regime for the heat to be carried away. Convection does not play a role either: Experiments were also performed in vacuum and helium atmosphere, which did not result in a significant difference. Since the

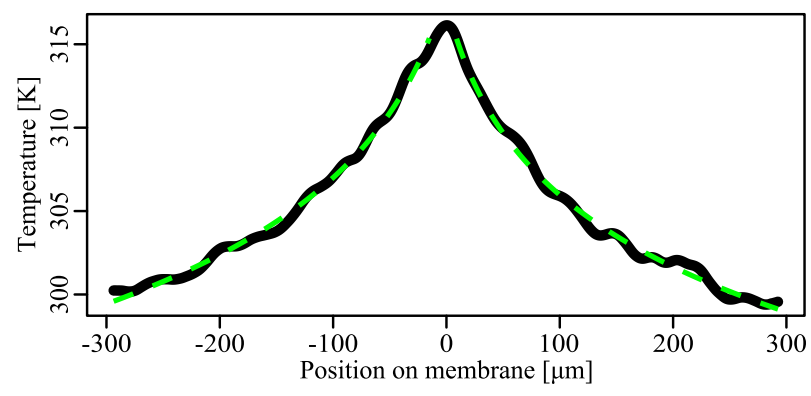

FIG. 2. (Color online) Temperature profile across a silicon membrane exploiting the temperature dependence of the transmissivity. The thin dashed lines show a fit using a simple model described in the text.

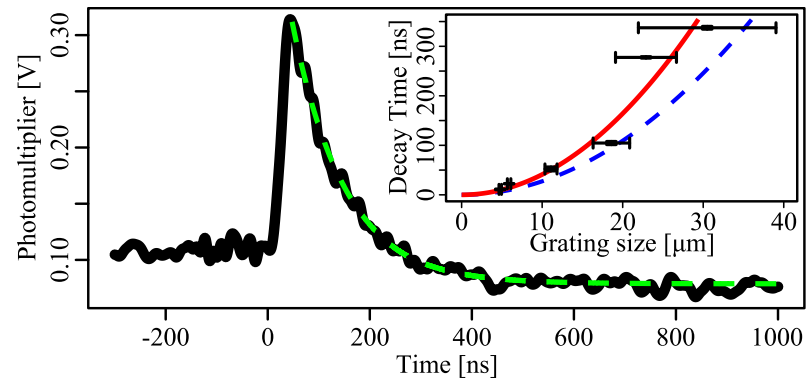

FIG. 3. (Color online) Temporal decay of a thermal transient grating of $18.6 \mu \mathrm{m}$ measured in first order of diffraction. The inset shows the expected $\Lambda^{2}$ dependence of $\tau_{\text {therm}}$, where the red solid line is a fit to the data and the blue dashed line represents the values from literature (Ref. 7).

result from Fig. 2 thus fits quite well to the quoted literature values, it is a proof of principle for the capability of this type of contactless temperature sensing.

As a complementary optical method, which demonstrates the excellent temporal resolution of Si membranes as temperature sensors, we have used diffraction from a transient laser-induced thermal grating; see Fig. 3. The grating was generated in the membrane by the interference of two laser beams at an angle $\theta$, obtained from a pulsed frequencydoubled Nd:yttrium aluminum garnet laser $(\lambda=532 \mathrm{~nm}$, pulse duration $13 \mathrm{~ns})$. The spacing $\Lambda=\lambda / 2 \sin (\theta / 2)$ of the interference fringes was varied between 5 and $30 \mu \mathrm{m}$. The temperature modulation in the membrane resulting from the interference pattern gives rise to a refractive index grating, the decay of which can be determined via diffraction of a cw probe beam $(\lambda=488 \mathrm{~nm})$. In Fig. 3 we show the intensity in the first order of diffraction, recorded with a fast photomultiplier. The steep rise at $t=0$ signals the buildup of the transient grating induced by the pump pulse; the subsequent exponential decrease reflects its decay by thermal diffusion.

The thermal conductivity $\kappa$ can be obtained by solving the one-dimensional heat equation for the given symmetry and is related to the relaxation time $\tau_{\text {therm }}$ of the spatial temperature modulation by

$$
\tau_{\text {therm }}=\frac{\rho c_{P} \Lambda^{2}}{4 \pi^{2} \kappa},
$$

where $\rho$ and $c_{P}$ are the density and specific heat of the sample, respectively. Due to the short distances in the micrometer range, over which the thermal diffusion takes place in this case, the relaxation times are in the submicrosecond regime. Measurements for varying lattice spacing, see inset in Fig. 3, confirm the $\Lambda^{2}$ dependence predicted by Eq. (2). Within the uncertainty of the measurement, our data agree with the values reported by Eichler et al. ${ }^{7}$ obtained for a $\mathrm{Si}$ film on glass, leading to an estimated value of $\kappa$ $=108 \pm 9 \mathrm{~W} / \mathrm{m} \mathrm{K}$. (As pointed out by Eichler et al., a detailed analysis requires taking contributions from photoexcited electrons and holes to the signals into account, which were neglected here.)

From Figs. 2 and 3 we conclude that Si membranes are a suitable tool to study temperature distributions and hence thermal processes. They should even lend themselves for a straightforward mapping of two-dimensional temperature patterns. That this is feasible indeed is shown in Fig. 4. In 

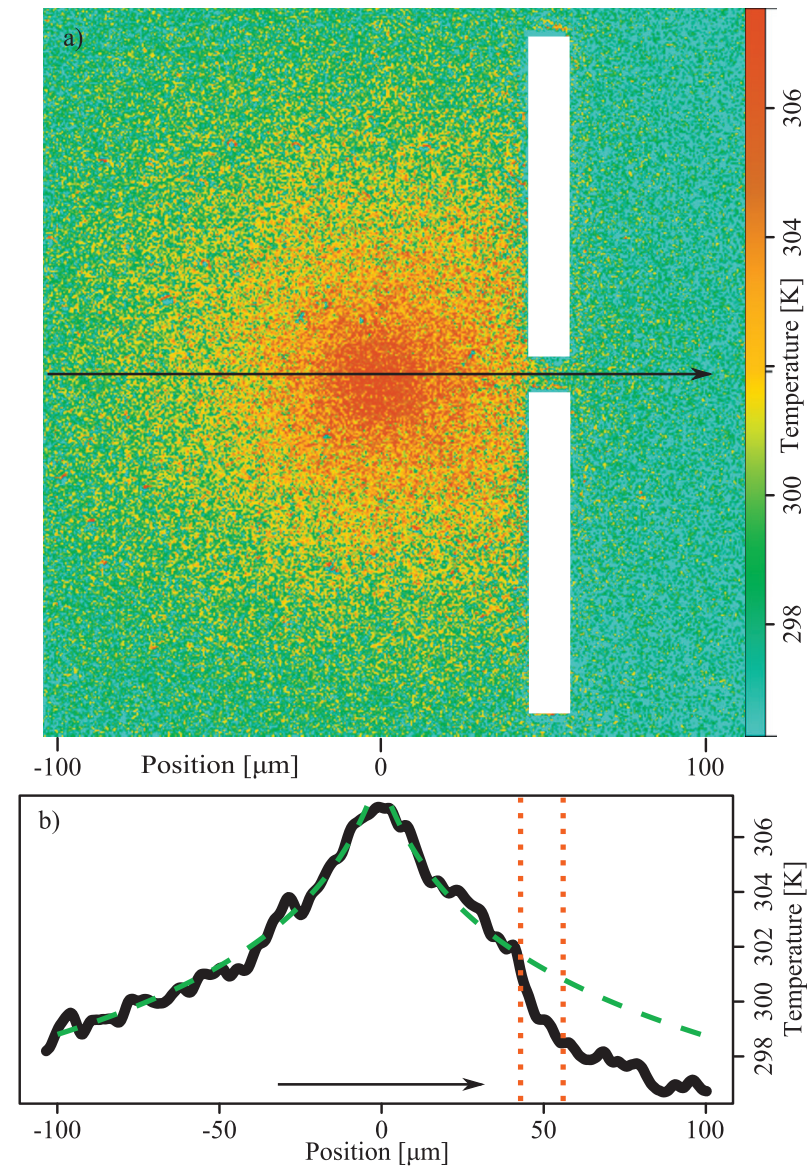

FIG. 4. (Color) (a) 2D heat pattern in a Si membrane measured at a fixed transmission wavelength with a CCD camera, over a $10 \mu \mathrm{m}$ wide bridge. (b) Temperature profile along the black arrow in Fig. 4(a). The two red dotted lines specify the position of the bridge and the green dashed solid lines are the fits to the data on the left wing.

this case, a Si membrane was placed under an optical microscope (objective magnification $10 \times$ ) and imaged with a charge-coupled device (CCD) camera with 16 bit resolution. Local heating was achieved like in Fig. 2 by a laser beam $\left(\lambda_{\text {heat }}=658 \mathrm{~nm}, a_{\text {heat }} \approx 10 \mu \mathrm{m}\right)$ focused to the center of the membrane. As light source for the transmission measurement we used a white light light-emitting diode, which could also be operated pulse-wise in order to gain temporal information. An optical laser-line filter $(\lambda=488 \mathrm{~nm})$ was placed in front of the CCD in order to protect it from stray light of the heating laser and to exclusively image the interesting wavelength of $488 \mathrm{~nm}$. The Si membrane sample was in this case modified by means of a focused ion beam. Two small gaps, each with a width of $10 \mu \mathrm{m}$ and a length of $100 \mu \mathrm{m}$ were machined next to each other so that a $10 \mu \mathrm{m}$ wide bridge was formed [in Fig. 4(a) on the right hand side]. It is obvious how the gaps in the membrane influence the thermal transport and-in contrast to Fig. 2 lead to an anisotropic temperature distribution. While the left wing in Fig. 4(b) closely follows the logarithmic decay found for an isotropic twodimensional (2D) system, the heat flow on the right hand side is associated with a much steeper temperature gradient across the bridge due to the confined geometry [Fig. 4(b)]. "Behind" the bridge it again follows the logarithmic dependence from Eq. (1).

Structuring of Si membranes with FIB or other high resolution lithography together with the presented method to derive temperature distributions opens the way for more complex applications like a micro-or "nanocalorimeter." The temperature profile around a small object placed onto the membrane could be used for measuring the specific heat of the object.

\section{SUMMARY}

In conclusion, the results presented here show that thin $\mathrm{Si}$ membranes are a promising material for investigating thermal properties, in particular when small length and time scales as well as high sensitivity are needed. The all-optical technique introduced here bears important advantages, allowing a very fast, high resolution 2D and contact-free mapping of temperature distributions without disturbing the system by electrical contacts.

\section{ACKNOWLEDGMENTS}

We thank Professor Dr. J. Boneberg, Professor Dr. T. Dekorsy, Dr. O. Schecker, R. Waitz, and F. Habel for discussions and contributions to the experiment. This work was supported by the Deutsche Forschungsgemeinschaft through SFB 767.

${ }^{1}$ D. G. Cahill, W. K. Ford, K. E. Goodson, G. D. Mahan, A. Majumdar, H. J. Maris, R. Merlin, and S. R. Phillpot, J. Appl. Phys. 93, 793 (2003).

${ }^{2}$ G. Jellison and H. Burke, J. Appl. Phys. 60, 841 (1986).

${ }^{3}$ J. Butschke, A. Ehrmann, E. Haugeneder, M. Irmscher, R. Kaesmaier, K. Kragler, F. Letzkus, H. Loeschner, J. Mathuni, I. W. Rangelow, C. Reuter, F. Shi, and R. Springer, Proc. SPIE 3665, 20 (1999).

${ }^{4}$ R. Waitz, O. Schecker, and E. Scheer, Rev. Sci. Instrum. 79, 093901 (2008).

${ }^{5}$ D. E. Aspnes and A. A. Studna, Phys. Rev. B 27, 985 (1983).

${ }^{6}$ A. D. McConnell and K. E. Goodson, Thermal Conduction in Silicon Micro- and Nanostructures (Begell House, Redding, CT, 2005).

${ }^{7}$ H. Eichler, F. Massmann, E. Biselli, K. Richter, M. Glotz, L. Konetzke, and X. Yang, Phys. Rev. B 36, 3247 (1987). 